\title{
Circadian gene hCLOCK contributes to progression of colorectal carcinoma and is directly regulated by tumor-suppressive microRNA-124
}

\author{
JIA-ZI YU ${ }^{1,2}$, NING SUN $^{3}$, YI-BING BEI ${ }^{1}$, XIAO-BO LI ${ }^{3}$, CHAO LU $^{3}$ and LU-CHUN HUA ${ }^{1}$ \\ ${ }^{1}$ Department of Surgery, Huashan Hospital, Fudan University, Shanghai 200040; \\ ${ }^{2}$ Department of Surgery, Ningbo Medical Center Lihuili East Hospital, Ningbo 315000; ${ }^{3}$ Department of Physiology \\ and Pathophysiology, Shanghai Medical College, Fudan University, Shanghai 200032, P.R. China
}

Received July 25, 2016; Accepted May 3, 3017

DOI: $10.3892 / \mathrm{mmr} .2017 .7596$

\begin{abstract}
An abundance of studies has demonstrated that disruption of circadian rhythms is one of the factors that may contribute to the initiation and development of human colorectal carcinomas (CRCs). Recently, microRNA-124 has been demonstrated to suppress tumor growth or metastasis of CRCs. However, the mechanisms of cross-talk between microRNA-124 (miR-124) and circadian rhythms in the regulation of CRCs are poorly understood. The present study demonstrated that the protein expression levels of human circadian locomoter output cycles protein kaput (hCLOCK) is significantly increased, while miR-124 is attenuated in high-grade human CRC tissues and in the more invasive colorectal cancer cell lines SW620 and LOVO. It was further demonstrated that hCLOCK is a direct target of miR-124. Upregulation of miR-124 significantly inhibited hCLOCK expression in LOVO cells, and consequently inhibited its promoting effects on the proliferation and migration of LOVO cells. In conclusion, these data revealed that hCLOCK serves an enhancing role, whereas mir-124 serves a suppressive role, in human CRC. Attenuation of miR-124, of which hCLOCK is a direct target, leads to increased hCLOCK expression and disruption of circadian rhythms in CRC.
\end{abstract}

\section{Introduction}

Living organisms on the earth exhibit circadian rhythms with a 24-h alternating pattern, allowing them to adapt to the daily cycle of light and dark and change of seasons (1). At the molecular level, circadian rhythms are generated by a set of

Correspondence to: Dr Lu-Chun Hua, Department of Surgery, Huashan Hospital, Fudan University, 21 Wulumuqi Road, Shanghai 200040, P.R. China

E-mail: drhua@126.com

Key words: circadian gene, hCLOCK, colorectal carcinoma, miRNA-124 clock genes and proteins, which include Aryl hydrocarbon receptor nuclear translocator-like protein 1, human circadian locomoter output cycles protein kaput (hCLOCK) and period circadian protein homolog (PER)1, 2 and 3. These clock genes work in accurate feedback loops and keep the levels of certain mRNAs and proteins oscillating throughout the $24 \mathrm{~h}$ period (2). It has been demonstrated that $>10 \%$ of all genes in the mammalian genome are under the regulation of circadian genes (3). As core circadian genes regulate gene expression associated with apoptosis, cell cycle and other signaling pathways in cells, in turn, they also regulate many physiological functions such as body temperature, blood pressure, immune responses and hormone biosynthesis (4). Therefore, these genes may be important in maintaining the regular biological status of the organism. There is increasing evidence indicating that dysfunction of hCLOCK links to the pathogenesis of cancer. For example, attenuation of all three PER genes may promote the tumorigenesis of breast cancer (5). In contrast, the expression of hCLOCK is upregulated in breast cancer tissues when compared to adjacent normal tissues or healthy ones (1). There is evidence that ablation of PER2 leads to the development of malignant lymphomas (6). There is also evidence demonstrating that hCLOCK is involved in pathogenesis of colorectal carcinomas (CRCs). In our previous studies, it was demonstrated that the expression level of human (h)PER2 was significantly reduced in human CRC tissues than in paired surrounding non-cancerous tissues. Deregulation of hPER2 associated with patient age, histological grade and TNM stage (7). In contrast, higher levels of hCLOCK expression were observed in human CRC tissues when compared with paired non-cancerous ones. hCLOCK expression was significantly higher in late-stage Dukes' grade tumors, or poorly differentiated, and in $64.3 \%$ of tumor cases with lymph node metastasis (8).

Although mammalian circadian rhythms have been extensively studied in recent years, most of them were based on post-translational modification or negative regulation at the transcriptional level (9-11). Several groups have focused research attention on microRNAs (miRNAs/miRs), a class of small noncoding RNAs that serve essential roles in post-transcriptional regulation of gene expression (12-13). 
As they target tumor suppressor genes or oncogenes, alterations of miRNAs have also been reported in the initiation and progression of CRCs. For example, miR-124 is frequently reported to be dysregulated in CRCs and serves a role of suppressing CRCs by targeting specific genes such as signal transducer and activator of transcription 3 (STAT3), structural maintenance of chromosomes protein 4 and RelA-associated inhibitor (14-17). However, the mechanisms of cross-talk between miR-124 and circadian genes in regulation of CRCs are poorly understood.

The present study demonstrated that the expression level of hCLOCK, a core circadian gene, is significantly increased in high-grade human CRCs tissues. In contrast, miR-124 is similarly attenuated in these tissues samples. In the LOVO CRC cell line, upregulation of miR-124 significantly inhibited expression of hCLOCK, and consequently inhibited invasion and proliferation, while at the same time promoting apoptosis of LOVO cells. Furthermore, it was revealed that miR-124 deregulated hCLOCK by directly targeting its 3'-untranslated region (3'-UTR). In conclusion, the present study demonstrated that the attenuation of miR-124 contributed elevated hCLOCK protein expression in high grade CRCs tissue, and promoted progression of human CRCs.

\section{Materials and methods}

Clinical samples. Resected CRC samples and paired non-cancerous tissues (males, 28; females, 22; $n=50$ ) were obtained from patients undergoing surgery for colorectal cancer in Huashan Hospital (Shanghai, China). All patients had a definitive pathological diagnosis and received neither chemotherapy nor radiotherapy prior to surgery. Following surgical removal, the samples were preserved in liquid nitrogen immediately and then moved to $-80^{\circ} \mathrm{C}$ for long term storage. The age of the patients, tumor site, tumor type, histological grade and TNM stage according to American Joint Committee on Cancer classification were recorded (18). Written informed consent was obtained from all patients used in the present study.

Immunohistochemical staining. Tissues were fixed in formalin, embedded in paraffin and cut into $4-\mu \mathrm{m}$ sections. Following deparaffinization, sections were rehydrated and subjected to antigen retrieval by microwaving in $0.01 \mathrm{M}$ sodium citrate $(\mathrm{pH} 6)$ for $10 \mathrm{~min}$. Sections were incubated at $4^{\circ} \mathrm{C}$ overnight with monoclonal antibodies against hCLOCK (\#5157; 1:100; Cell signaling Technology, Inc., Danvers, MA, USA). After a brief wash with PBS, horseradish peroxidase-conjugated secondary antibody (\#A31460, 1:500; Thermo Fisher Scientific, Inc., Waltham, MA, USA) was added for $60 \mathrm{~min}$ at $37^{\circ} \mathrm{C}$. After several washes with PBS, staining was achieved using 3,3'-diaminobenzidine for 5 10 min. Finally, slides were counterstained with Mayer's hemalum and mounted. Protein staining was evaluated under a light microscope at x400 magnification. Staining intensity was scored manually by two independent experienced pathologists as follows: $0=$ no staining, $1=$ weak staining, $2=$ moderate staining and $3=$ strong staining. Tumor cells in five fields were randomly selected and scored based on the percentage of positively stained cells $(0-100 \%)$. The final score was calculated by multiplying the intensity score with the percentage of positive cells.

Cell culture. The SW620, SW480, LOVO and HT-29 CRC cell lines were purchased from the Chinese Academy of Sciences Institute (Shanghai, China). The cell lines were cultured in Dulbecco's modified Eagle's medium (Gibco; Thermo Fisher Scientific, Inc.) supplemented with $10 \%$ fetal bovine serum (Invitrogen; Thermo Fisher Scientific, Inc.).

miRNA target prediction. TargetScan (19) (http://www. targetscan.org), PicTar (20) (http://www.pictar.org/) and miRNA.org (21) (http://www.microrna.org) were used to predict the interaction between miRNAs and the hCLOCK 3'-UTR.

Total RNA extraction and reverse transcription-quantitative polymerase chain reaction $(R T-q P C R)$. Preparation of total RNA from CRC tissue samples and cultured cell lines was performed using TRIzol reagent (Invitrogen; Thermo Fisher Scientific, Inc.). The amount of total RNA was determined by ultraviolet spectrophotometry. Total RNA was reverse transcribed using a MicroRNA Reverse Transcription kit (Applied Biosystems; Thermo Fisher Scientific, Inc.) and a miR-124-specific stem-loop primer purchased from GeneChem Inc. (Daejon, Korea). The primer sequence for miR-124 was as follows: 5'-GTCGTATCCAGTGCAGGGTCCGAGGTATTC GCACTGGATACGACGGCATTC-3'. The PCR reactions containing SYBR-green (Toyobo Co., Ltd., Osaka, Japan) for mature miR-124 were amplified on a Corbett Real Time PCR machine (Bio-Rad, USA). The reaction mixtures for PCR contained $1 \mu \mathrm{l} \mathrm{cDNA,} 4 \mu \mathrm{l} 10 \mathrm{mmol} / \mathrm{l}$ primers $(2 \mu \mathrm{l}$ forward primers, $2 \mu \mathrm{l}$ reverse primers), $10 \mu \mathrm{l}$ real-time PCR Master Mix (Toyobo Co., Ltd., Osaka, Japan), and $5 \mu \mathrm{H}_{2} \mathrm{O}$, into a final volume of $20 \mu \mathrm{l}$, and run under the following conditions: One cycle at $95^{\circ} \mathrm{C}$ for $5 \mathrm{~min}$, and 40 cycles at $95^{\circ} \mathrm{C}$ for $5 \mathrm{sec}, 60^{\circ} \mathrm{C}$ for $30 \mathrm{sec}$ and $72^{\circ} \mathrm{C}$ for $5 \mathrm{sec}$. The sequences for qPCR primers were as follows: Forward, 5'-GCTAAGGCACGCGGTG-3' and reverse, 5'-CAGTGCAGGGTCCGAGGT-3'. The relative amounts of miR-124-3p were measured with the $2^{-\Delta \Delta C q}(22)$ method.

Western blot analysis. The cells were lysed with radioimmunoprecipitation assay buffer (Cell Signaling Technology, Inc.) and $1 \mathrm{mM}$ phenylmethanesulfonyl fluoride. The lysates were incubated at $4^{\circ} \mathrm{C}$ for $10 \mathrm{~min}$ and centrifuged at $13,400 \mathrm{x}$ g at $4^{\circ} \mathrm{C}$ for $15 \mathrm{~min}$. Equal amounts of lysate ( $25 \mu \mathrm{g}$ protein per lane) were separated by $12 \%$ SDS-PAGE and transferred to a polyvinylidene fluoride membrane (EMD Millipore, Billerica, MA, USA). The membranes were blocked in $5 \%$ non-fat skim milk/PBS with Tween-20 at room temperature for $2 \mathrm{~h}$, and probed with a primary antibody against hCLOCK (Cell signaling Technology, Inc.; \#5157; 1:1,000) at room temperature for $2 \mathrm{~h}$. The membranes were then incubated for $2 \mathrm{~h}$ at room temperature with a horseradish peroxidase-conjugated secondary antibody (Thermo Fisher Scientific, Inc.; \#A31460; 1:10,000), followed by incubation with enhanced chemiluminescence western blot detection reagent (GE Healthcare, Chicago, IL, USA). The hCLOCK primary antibody was purchased from Santa Cruz Biotechnology, Inc. (Dallas, TX, USA). 


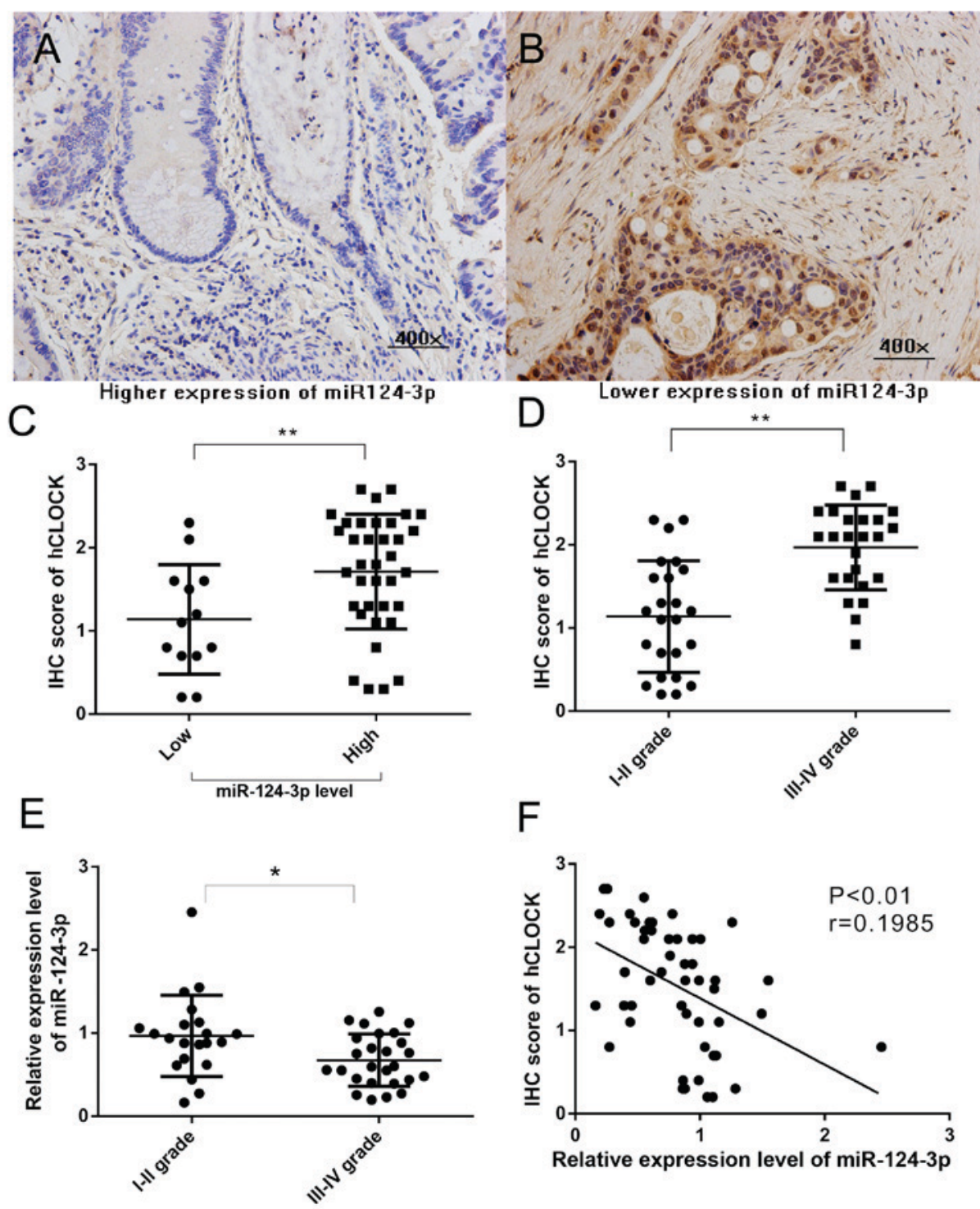

Figure 1. Association of miR-124-3p expression with hCLOCK expression in CRC tissues. (A and B) hCLOCK expression level in the CRC tissues with different miR-124-3p levels detected by immunohistochemistry staining. (C) hCLOCK level in CRC tissues with high mir-124-3p expression was significantly increased compared with CRC tissues with low miR-124-3p. (D) hCLOCK expression was significantly increased in late-stage CRC patients (n=50), (E) miR-124-3P expression was significantly lower in late-stage CRC patients. (F) A significant negative correlation was observed between mir-124-3p and hCLOCK level in CRC tissues. Data are presented as the mean \pm standard deviation $(n=50)$. ${ }^{*} \mathrm{P}<0.05,{ }^{* *} \mathrm{P}<0.01$. hCLOCK, human circadian locomoter output cycles protein kaput; CRC, colorectal cancer; miR, microRNA; IHC, immunohistochemistry.

Lentiviral vector construction and infection. A GV258 lentiviral vector, GV214 vector containing miR-124 precursor sequences and a negative control (NC) were purchased from GeneChem, Inc. The human miR-124 precursor gene was PCR amplified from normal genomic DNA, and cloned into the GV258 lentiviral vector or GV214 for ectopic expression of miR-124. Primers used for amplification were 5'-AGCTGT ACAAGTAAGTTCTTATTCCATCTTCTACC-3' (forward) and 5'-AGCTGTACAAGTAAGTTCTTATTCCATCTTCTA CC-3' (reverse: with NheI site). LOVO cells were infected with miR-NC or mir-124 overexpression viruses and selected by puromycin, and were designed as miR-124 LOVO and miR-NC LOVO, respectively.

Dual-luciferase reporter construction. GV306 vectors with a fragment of the 3'-UTR of hCLOCK gene (NM_004898) containing the recognition site for miR-124-3p at nucleotide position (from nt 930 to nt1501, with XbaI sites), the corresponding site-directed mutant containing the mutated seed sequence and the NC were purchased from GeneChem, Inc. The fragment was constructed via a generate gene synthesis service (Shanghai GeneChem Co., Ltd., Shanghai, China). The corresponding site-directed mutant containing the mutated seed sequence (5'-GTGCCTT-3' to 5'-CGTAGAG-3') was also generated. The wild type and mutated hCLOCK 3'-UTR fragments were then cloned into pmirGLO Dual-Luciferase miRNA Target Expression Vector (Promega Corporation, Madison, WI, USA) at XbaI/XbaI sites and termed Luc-hCLOCK-wt and Luc-hCLOCK-mt, respectively. A luciferase vector without hCLOCK 3'-UTR (Luc-hCLOCK-ctl) was used as experimental control. All constructs were confirmed by DNA sequencing. 


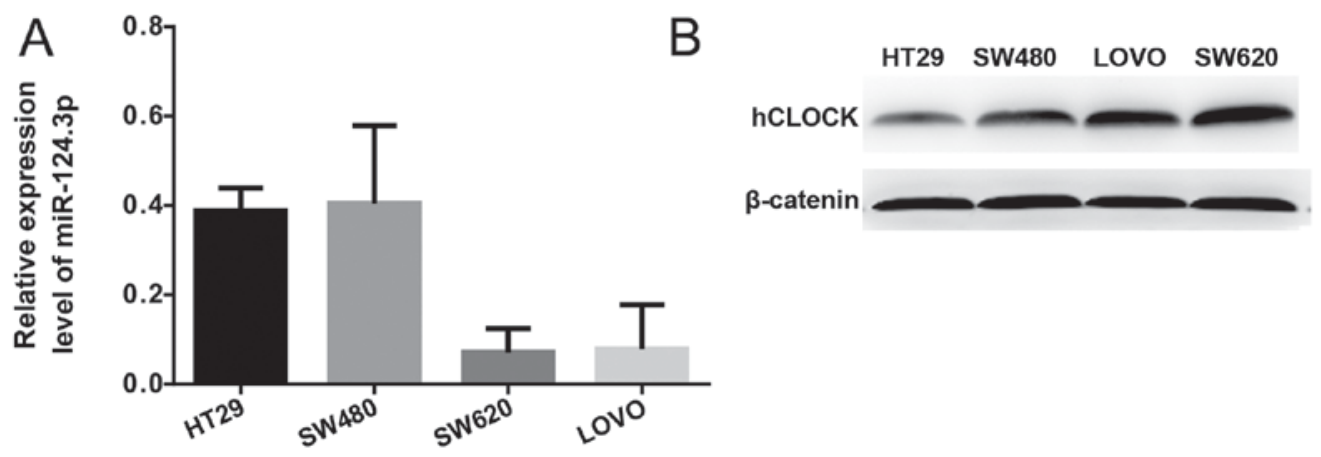

Figure 2. Expression level of miR-124-3p in human CRC cell lines. (A) Reverse transcription-quantitative polymerase chain reaction analysis of miR-124-3p expression in four CRC cell lines. Data are presented as the mean \pm standard deviation $(\mathrm{n}=3)$. (B) Western blot analysis was used to detect hCLOCK protein expression levels in four CRC cell lines. $\beta$-catenin served as the loading control. HT29 and SW480, less invasive cell lines; LOVO and SW620, more invasive ones. CRC, colorectal cancer; miR, microRNA; hCLOCK, human circadian locomoter output cycles protein kaput.

Luciferase assay. 293T cells were seeded overnight in antibiotic-free complete medium at $5 \times 10^{4}$ cells/well on a 24 -well plate. The cells were transfected with $2 \mu \mathrm{g}$ miR-124-3p or miR-NC and $400 \mathrm{ng}$ Luc-hCLOCK-NC, Luc-hCLOCK-wt or Luc-hCLOCK-mt for $48 \mathrm{~h}$. The co-transfection procedure for miRNAs and luciferase reporter constructs was performed using Lipofectamine 2000 reagent (Invitrogen; Thermo Fisher Scientific, Inc.). A luciferase activity assay was performed using the Dual-Luciferase Reporter Assay system (Biotime, Shanghai, China) according to the manufacturer's instructions. The levels of firefly luciferase activities were obtained by normalizing to Renilla luciferase activities and relative to a control.

In vitro cell proliferation assays. Cell proliferation was determined by CellCounting kit-8 (Dojindo MolecularTechnologies, Inc., Kumamoto, Japan) assay. In brief, miR-124-LOVO, miR-NC-LOVO and LOVO cells were seeded into 96-well plates at an density of $1 \times 10^{4}$ cells per well. After 12, 24, 36, 48 and $72 \mathrm{~h}, 10 \mu \mathrm{l}$ kit reagent was added to each well, and $2 \mathrm{~h}$ later all plates were scanned by a microplate reader (Thermo Fisher Scientific, Inc.) at $450 \mathrm{~nm}$. Cell proliferation was calculated on the basis of absorbency.

In vitro invasion assays. Cell invasion was analyzed by a Transwell Permeable Supports system with $12-\mu \mathrm{m}$ pores (Corning Incorporated, Corning, NY, USA). Cells were observed under an inverted phase microscope. Cells $\left(2-3 \times 10^{5}\right)$ were seeded onto upper inserts with a Matrigel-coated membrane (BD Biosciences, Franklin Lakes, NJ, USA). Cells were seeded in serum-free medium and transferred to $10 \%$ serum media for $48 \mathrm{~h}$. After removal of the non-invading cells, the remaining cells were fixed, stained, eluted by $30 \%$ acetic acid, scanned by a microplate reader (Thermo Fisher Scientific, Inc.) at $450 \mathrm{~nm}$ and finally calculated on the basis of absorbency.

Evaluation of cell apoptosis. Annexin V-fluorescein isothiocyanate (FITC)/propidium iodide (PI) staining was performed to quantify cell apoptosis. Briefly, cells were collected, washed in cold PBS twice and suspended in binding buffer at $1 \times 10^{6}$ cells $/ \mathrm{ml}$. Cells were then stained with Annexin V-FITC and PI according to the manufacturer's protocol. Cell apoptosis was detected by a FACSVerse flow cytometer, equipped with FACSuite software version 1.0 (both from BD Biosciences, Franklin Lakes, NJ, USA).

Statistical analysis. All values are expressed as the mean \pm standard error. Results were analyzed by Student's t-test or one-way analysis of variance, followed by Fisher's least significant difference test, as appropriate. Pearson's correlation test was used to assess the correlation between two variables. Statistical analysis was performed using SPSS 11.0 (SPSS, Inc., Chicago, IL, USA). $\mathrm{P}<0.05$ was considered to indicate a statistically significant difference.

\section{Results}

Attenuation of miR-124-3p leads to overexpression of hCLOCK in CRCs tissues of clinical patients, and is correlated with patients of the late stage CRC. Our previous study reported that increased hCLOCK expression is observed in human CRC tissues compared with paired non-cancerous tissues (8). To evaluate the association between miR-124-3p level and hCLOCK expression in CRCs, hCLOCK expression in CRC tissues with different miR-124-3p expression levels was analyzed. miR-124-3p expression was defined as low if the relative level in the CRC tissue was lower than the paired non-cancerous tissue, and high if the relative miR-124-3p level was higher than the paired non-cancerous tissue. The hCLOCK level in CRC tissues with high miR-124-3p were significantly decreased compared with those CRCs with low miR-124-3p expression ( $\mathrm{P}<0.01$; Fig. 1A-C). The present study confirmed that a higher level of hCLOCK expression was significantly correlated with patients with late stage CRC $(\mathrm{P}<0.001$; Fig. 1D). Furthermore, the results demonstrated that downregulated expression of miR-124-3p was significantly correlated with late TNM stage in patients (Fig. 1E). Significant negative correlations were observed between miR-124-3p level and hCLOCK expression $(r=0.1985, \mathrm{P}<0.01)$ in CRC tissues (Fig. 1F).

Expression level of miR-124-3p is negatively correlated with the protein expression level of hCLOCK in human CRC cells. Protein expression levels of hCLOCK and the mRNA expression levels of miR-124-3p in the SW620, SW480, HT29 


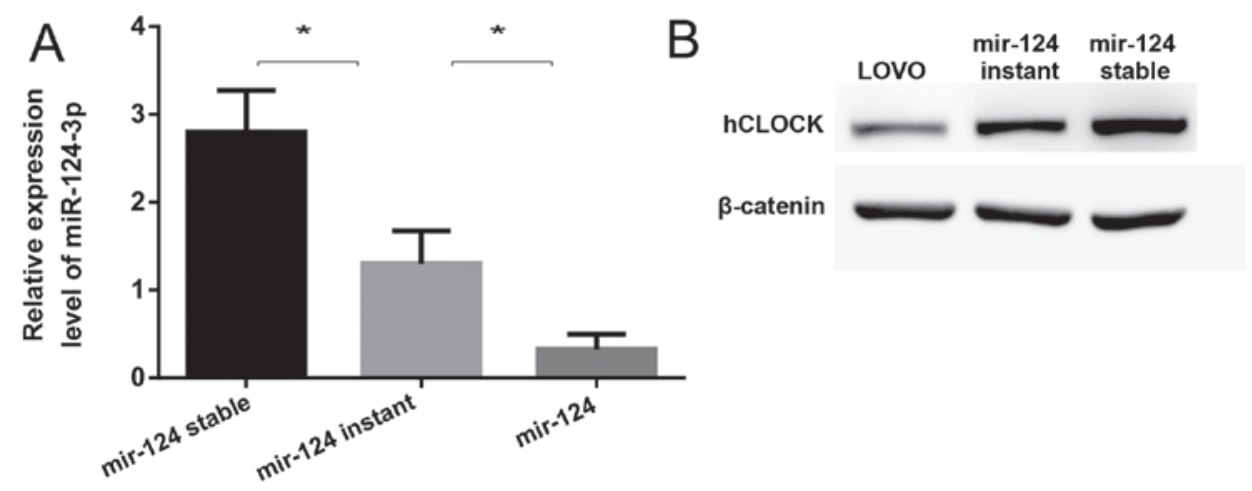

Figure 3. Upregulation of miR-124 contributes to the decreased protein expression level of hCLOCK in colorectal cancer cells. (A) miR-124-stable LOVO cells were generated by stable transfection with miR-124 over-expression lentivirus and expressed miR-124-3p stably. miR-124-instant LOVO cells were generated by transient transfection with plasmids and expressed mir-124-3p instantly. Reverse transcription-quantitative polymerase chain reaction was used to detect the expression of miR-124-3p in these three types of cells. Data are presented as the mean \pm standard deviation ( $\mathrm{n}=3$ ). (B) Western blotting analysis was used to detect hCLOCK protein expression levels. $\beta$-catenin served as the loading control. ${ }^{~} \mathrm{P}<0.05$. miR, microRNA; hCLOCK, human circadian locomoter output cycles protein kaput.
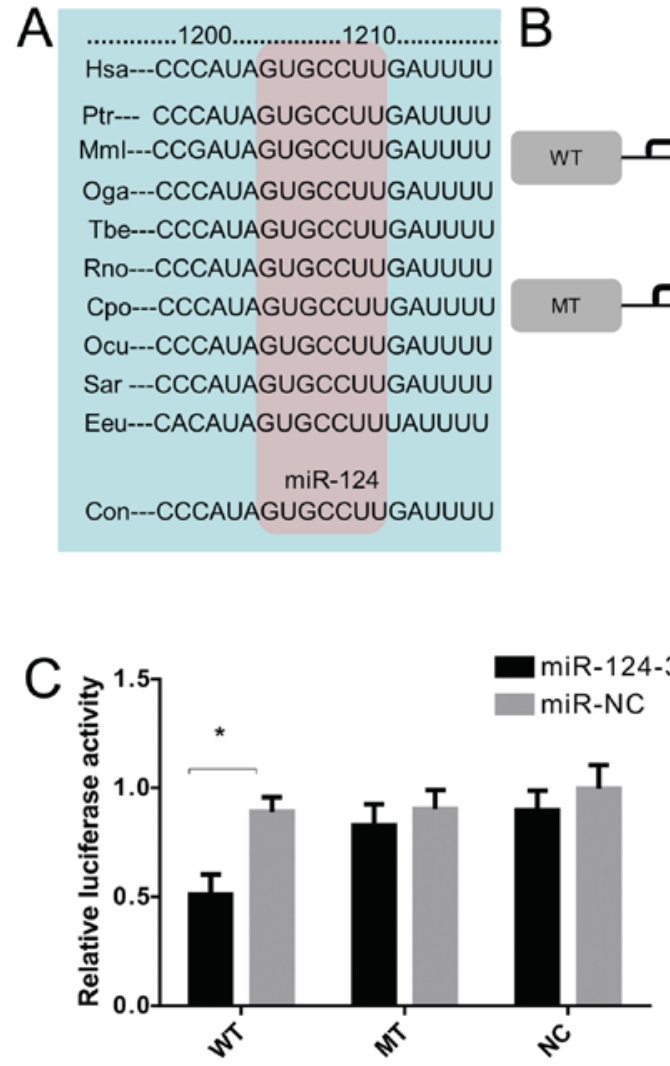

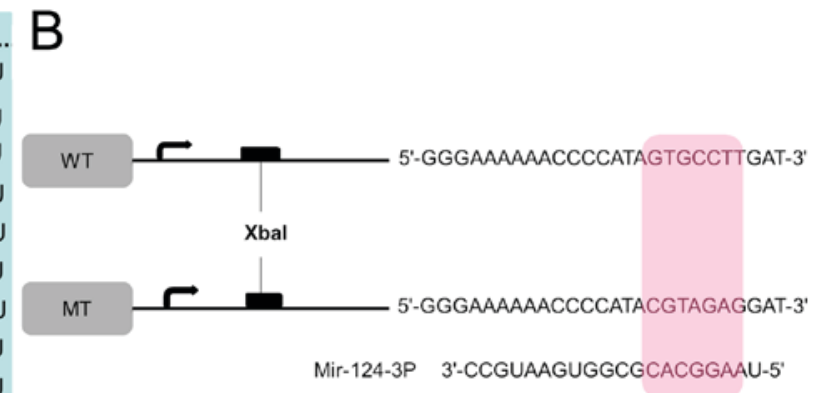

Mir-124-3P 3'-CCGUAAGUGGCGCACGgAAU-5'

D

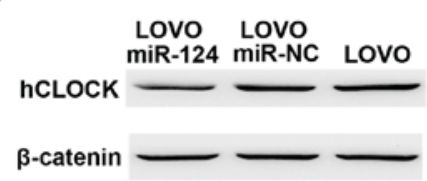

Figure 4. hCLOCK is a direct target of miR-124-3p. (A) The miR-124 targeting sequence in the 3'-UTR of hCLOCK is conserved among different species. (B) Either WT or MT hCLOCK 3'-UTR was cloned downstream of the luciferase reporter gene; the nucleotides of recognition site for miR-124-3p are highlighted in pink. (C) The interactions between miR-124 and the 3'-UTRs of hCLOCK were tested by luciferase reporter assays. Data are presented as the mean \pm standard deviation $(n=3)$. ${ }^{*} \mathrm{P}<0.05$. (D) Western blotting analysis examining hCLOCK protein expression. $\beta$-catenin served as the loading control. WT, wild-type; NC, negative control; MT, mutant; miR, microRNA; hCLOCK, human circadian locomoter output cycles protein kaput.

and LOVO human CRC cell lines were examined by western blotting and RT-qPCR, respectively. It was demonstrated that miR-124-3p expression was significantly increased in more invasive CRC cell lines (SW620 and LOVO) compared with less invasive CRC cell lines (SW480 and HT29; Fig. 2A). In contrast, the protein expression levels of hCLOCK was increased in less invasive CRC cell lines compared with more invasive ones (Fig. 2B).
Upregulation of miR-124 contributes to suppression of hCLOCK expression in human CRC cells. Using three widely used microRNA target prediction databases (TargetScan, PicTar and miRNA.org), miR-124-3p was predicted to putatively bind to the 3'-UTR of hCLOCK. To confirm whether miR-124-3p suppresses the expression of hCLOCK, the miR-124 precursor was transfected into LOVO cells by lentiviral vectors or plasmids, representing mir-124 stable 

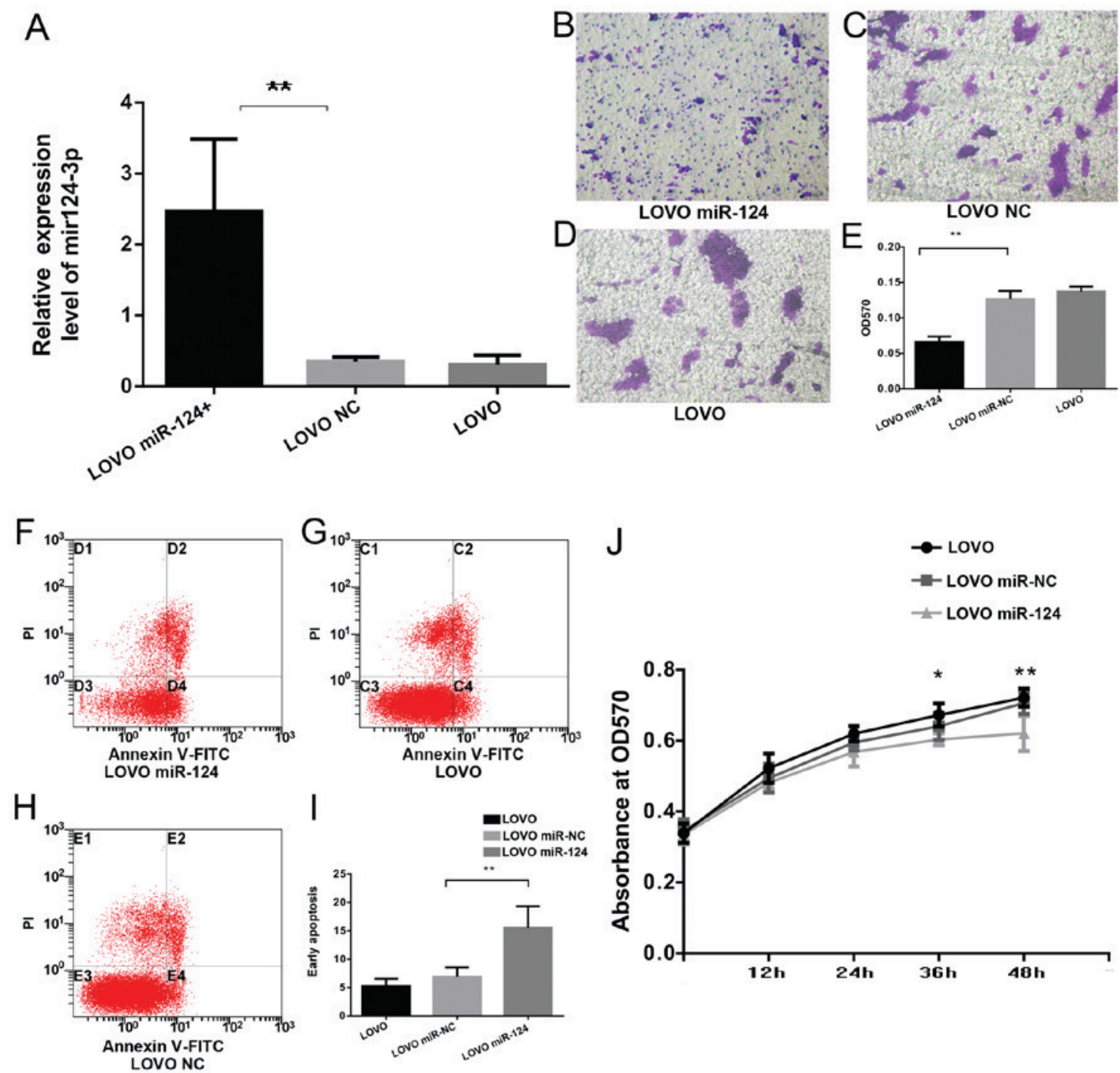

Figure 5. miR-124-3p induces apoptosis and inhibits invasion and proliferation of CRC cells. (A) Reverse transcription-quantitative polymerase chain reaction was used to detect the expression of miR-124-3p. (B-D) Cell invasion was analyzed by a Transwell Permeable Supports system in (B) LOVO miR-142, (C) LOVO NC and (D) LOVO cells, and (E) the results were quantified. Annexin V/PI staining of (F) LOVO miR-142, (G) LOVO NC and (H) LOVO cells, and (I) quantification of apoptosis. (J) Cell proliferation assay examining proliferation rate of LOVO cells transfected with miR-124 or miR-NC, and LOVO cells without transfection. Data are presented as the mean \pm standard deviation $(n=3)$. ${ }^{*} \mathrm{P}<0.05$ and ${ }^{* * *} \mathrm{P}<0.01, \mathrm{LOVO}$ miR-142 vs. LOVO NC or LOVO cells, or as indicated. miR, microRNA; hCLOCK, human circadian locomoter output cycles protein kaput; NC, negative control; FITC, fluorescein isothiocyanate; PI, propidium iodide; OD, optical density.

expression (miR-124-stable) and mir-124 instant expression (miR-124-instant), respectively. It was demonstrated that the expression of miR-124-3p was significantly increased in miR-124-stable and miR-124-instant cells compared to LOVO cells (Fig. 3A), while hCLOCK expression was significantly suppressed in both cell lines (Fig. 3B). Notably, it was revealed that the higher the miR-124-3p expression was, the lower the hCLOCK protein level was in these cells (Fig. 3).

hCLOCK is a direct target of miR-124-3p. To examine whether hCLOCK is a direct target of miR-124-3p, a miR-124-3p based luciferase assay was performed to observe whether miR-124-3p binds to the 3'-UTR of hCLOCK mRNA. The miR-124 targeting sequence in 3'-UTR of hCLOCK was identified to conserved among different species (Fig. 4A). Wild-type and mutant hCLOCK 3'-UTR were cloned downstream of the luciferase reporter gene (Fig. 4B). The results demonstrated that miR-124-3p directly bound to the hCLOCK 3'-UTR and significantly reduced the luciferase activities, while cells with mutant hCLOCK 3'-UTR exhibited higher luciferase activities (Fig. 4C). Furthermore, western blot analysis demonstrated that exogenous miR-124-3p expression suppressed endogenous hCLOCK protein levels in LOVO cells (Fig. 4D). Taken together, these results indicated that hCLOCK is a direct target for miR-124-3p in CRC cells.

miR-124-3p induces apoptosis and inhibits invasion and proliferation of CRC cells. As presented in Fig. 5A, transfection of the miR-124 lentiviruses caused a significant increase in the expression of miR-124-3p in LOVO cells relative to LOVO cells transfected with mir-NC viruses (LOVO miR-NC) and non-transfected LOVO cells (LOVO). Compared with LOVO-miR-NC and LOVO cells, the invasion ability was significantly inhibited in LOVO cells transfected with miR-124 
(LOVO miR-124; Fig. 5B-E). miR-124-3p transfection significantly increased the apoptosis rate (LOVO miR-124, $15.54 \pm 3.81 \%$ vs. LOVO miR-NC, $6.91 \pm 1.63 \%$ vs. LOVO. $5.27 \pm 1.30 \%$; Fig. 5F-I). Furthermore, the proliferation of LOVO cells was significantly inhibited 36 and $48 \mathrm{~h}$ after transfection with miR-124-3p (Fig. 5J).

\section{Discussion}

CRC is the third most common cancer in men and the second in women worldwide (23). Despite improvement in therapeutic strategies for CRCs, including surgical technique and chemo-radiotherapy, the prognosis remains extremely poor (24). Further studies on the molecular mechanisms underlying CRC initiation and progression are crucial for developing effective and specific treatment strategies (25-26).

In our previous study, it was demonstrated that disruption of circadian rhythms, which are regulated by circadian genes, was one of the endogenous factors that contribute to CRC tumor initiation and progression $(7,8)$. As a core circadian gene, hCLOCK is highly involved in this process. However, the regulation mechanism of hCLOCK in CRC initiation and progression are poorly understood. The present study hypothesized that attenuation of miRNAs targeting hCLOCK would lead to decreased expression of hCLOCK in CRCs, thereby inhibiting CRC initiation and progression. After searching for miRNAs targeting hCLOCK through miRNA predicting databases, miR-124, which is one of the microRNAs extensively studied in CRCs, was identified to directly target the hCLOCK 3'-UTR. Increasing evidence has demonstrated that miR-124 is dysregulated in CRCs and serves a suppressive role for CRC by targeting specific genes (15-18). miR-124 is frequently silenced in CRCs, possibly due to hypermethylation of the miR-124 promoter region (27). mir-124 promoter hypermethylation may be one of the factors affecting the expression level of miR-124, and may be responsible for high expression of hCLOCK in CRCs.

Therefore, the present study aimed to examine the expression level of miR-124-3p and hCLOCK in human $\mathrm{CRC}$ tissues. The results indicated that the expression level of hCLOCK is significantly upregulated in the III/IV grade TNM stage CRCs tissues compared with I/II grade ones. In contrast, miR-124 was attenuated in the same tissue samples. The hCLOCK expression level in CRC tissues with high levels of mir-124-3p was significantly reduced, compared with CRC tissues with low levels of mir-124-3p. Significant negative correlations were demonstrated between miR-124-3p and hCLOCK expression levels in CRCs. The same experiments performed using human CRC cell lines also revealed the same phenomenon. The expression level of hCLOCK was significantly upregulated in the more invasive CRC cell lines SW620 and LOVO. In contrast, miR-124 was attenuated in less invasive CRC cell lines SW480 and HT29. Upregulation of miR-124 significantly inhibited the expression level of hCLOCK in LOVO cells. Furthermore, the impact of miR-124 on proliferation, invasion and apoptosis in CRC cells was examined. Upregulation of miR-124 significantly inhibited proliferation and invasion and induced apoptosis of LOVO cells. Furthermore, dual-luciferase assays indicated that hCLOCK is a direct target of miR-124.
In conclusion, the present study demonstrated that hCLOCK has an enhancing role miR-124 has a suppressive role in the progression of human CRCs. Furthermore, hCLOCK mRNA was a direct target of miR-124. Attenuation of miR-124 in CRCs contributed to the high hCLOCK expression, and finally promoted the progression of human CRCs. These findings expand the understanding of the underlying mechanisms of circadian rhythms in regulating human CRCs, and may provide a novel perspective for future clinical therapy of this type of cancer.

\section{Acknowledgements}

This work was supported by the Project for National Basic Science Personnel Training Fund (no. J1310009) and the National Natural Science Foundation of China (no. 81570771).

\section{References}

1. Hoffman AE, Yi CH, Zheng T, Stevens RG, Leaderer D, Zhang Y, Holford TR, Hansen J, Paulson J and Zhu Y: CLOCK in breast tumorigenesis: Genetic, epigenetic, and transcriptional profiling analyses. Cancer Res 70: 1459-1468, 2010.

2. Ko CH and Takahashi JS: Molecular components of the mammalian circadian Clock. Hum Mol Genet 15: R271-R277, 2006.

3. Storch KF, Lipan O, Leykin I, Viswanathan N, Davis FC, Wong WH and Weitz CJ: Extensive and divergent circadian gene expression in liver and heart. Nature 417, 78-83, 2002.

4. Benca R, Duncan MJ, Frank E, McClung C, Nelson RJ and Vicentic A: Biological rhythms, higher brain function, and behavior: Gaps, opportunities, and challenges. Brain Res Rev 62: 57-70, 2009.

5. Chen ST, Choo KB, Hou MF, Yeh KT, Kuo SJ and Chang JG: Deregulated expression of the PER1, PER2 and PER3 genes in breast cancers. Carcinogenesis 26: 1241-1246, 2005.

6. Fu L, Pelicano H, Liu J, Huang P and Lee C: The circadian gene period 2 plays an important role in tumor suppression and DNA damage response in vivo. Cell 111: 41-50, 2002.

7. Wang Y, Hua L, Lu C and Chen Z: Expression of circadian Clock gene human period2 (hPer2) in human colorectal carcinoma. World J Surg Oncol 9: 166, 2011.

8. Wang L, Chen B, Wang Y, Sun N, Lu C, Qian R and Hua L: hClock gene expression in human colorectal carcinoma. Mol Med Rep 8: 1017-1022, 2013.

9. Dibner C, Schibler U and Albrecht U: The mammalian circadian timing system: Organization and coordination of central and peripheral clocks. Ann Rev Physiol 72: 517-549, 2010.

10. Harms E, Kivimäe S, Young MW and Saez L: Posttranscriptional and posttranslational regulation of clock genes. J Biol Rhythms 19: 361-373, 2004.

11. Asher G, Gatfield D, Stratmann M, Reinke H, Dibner C, Kreppel F, Mostoslavsky R, Alt FW and Schibler U: SIRT1 regulates circadian clock gene expression through PER2 deacetylation. Cell 134: 317-328, 2008.

12. Bartel DP: MicroRNAs: Target recognition and regulatory functions. Cell 136: 215-233, 2009.

13. Long JM and Lahiri DK: Advances in microRNA experimental approaches to study physiological regulation of gene products implicated in CNS disorders. Exp Neurol 235: 402-418, 2012.

14. Wang MJ, Li Y, Wang R, Wang C, Yu YY, Yang L, Zhang Y, Zhou B,Zhou ZG and Sun XF: Downregulation of microRNA-124 is an independent prognostic factor in patients with colorectal cancer. Int J Colorectal Dis 28: 183-189, 2013.

15. Zhang J, Lu Y, Yue X, Li H, Luo X, Wang Y, Wang K and Wan J: MiR-124 suppresses growth of human colorectal cancer by inhibiting STAT3. PLoS One 8: e70300, 2013.

16. Jinushi T, Shibayama Y, Kinoshita I, Oizumi S, Jinushi M, Aota T, Takahashi T, Horita S, Dosaka-Akita H and Iseki K: Low expression levels of microRNA-124-5p correlated with poor prognosis in colorectal cancer via targeting of SMC4. Cancer Med 3: 1544-1552, 2014.

17. Liu K, Zhao H, Yao H, Lei S, Lei Z,Li T and Qi H: MicroRNA-124 regulates the proliferation of colorectal cancer cells by targeting iASPP. Biomed Res Int 2013: 867537, 2013. 
18. Edge SB, Byrd DR, Compton CC, Fritz AG, Greene FL and Trotti A: AJCC Cancer Staging Manual. 7th edition. New York, Springer, 2010

19. Agarwal V, Bell GW, Nam JW and Bartel DP: Predicting effective microRNA target sites in mammalian mRNAs. Elife 4: 2015.

20. Krek A, Grün D, Poy MN, Wolf R, Rosenberg L, Epstein EJ, MacMenamin P, da Piedade I, Gunsalus KC, Stoffel M and Rajewsky N: Combinatorial microRNA target predictions. Nat Genet 37: 495-500, 2005

21. Betel D, Wilson M, Gabow A, Marks DS and Sander C: The microRNA.org resource: targets and expression. Nucleic Acids Res 36 (Database Issue): D149-D153, 2008.

22. Livak KJ and Schmittgen TD: Analysis of relative gene expression data using real-time quantitative PCR and the 2(-Delta Delta C(T)) method. Methods 25: 402-408, 2001.

23. Ferlay J, Shin HR, Bray F, Forman D, Mathers C and Parkin DM: Estimates of worldwide burden of cancer in 2008: GLOBOCAN 2008. Int J Cancer 127: 2893-2917, 2010.
24. Venook AP, Niedzwiecki D, Lenz HJ, et al: CALGB/SWOG 80405: Phase III trial of irinotecan/5-FU/leucovorin (FOLFIRI) or oxaliplatin/5-FU/leucovorin (mFOLFOX6) with bevacizumab (BV) or cetuximab (CET) for patients with KRAS wild-type untreated metastatic adenocarcinoma of the colon or rectum [abstract]; 2014 American Society of Clinical Oncology Annual Meeting; 2014 May 30-June 3; Chicago, USA. Alexandria (VA): American Society of Clinical Oncology, 2014.

25. Cancer Genome Atlas Network: Comprehensive molecular characterization of human colon and rectal cancer. Nature 487: 330-337, 2012.

26. Fearon ER: Molecular genetics of colorectal cancer. Annu Rev Pathol 6: 479-507, 2011

27. Harada T, Yamamoto E, Yamano HO, Nojima M, Maruyama R, Kumegawa K, Ashida M, Yoshikawa K, Kimura T, Harada E, et al: Analysis of DNA methylation in bowel lavage fluid for detection of colorectal cancer. Cancer Prev Res (Phila) 7: 1002-1010, 2014. 